\title{
Robust Difference Scheme for the Cauchy Problem for a Singularly Perturbed Ordinary Differential Equation
}

\author{
Lidia P. Shishkina ${ }^{a}$ and Grigory I. Shishkin ${ }^{a}$ \\ ${ }^{a}$ Institute of Mathematics and Mechanics, Russian Academy of Sciences \\ Yekaterinburg 620990, GSP-384, Russia \\ E-mail(corresp.): Lida@convex.ru \\ E-mail: shishkin@imm.uran.ru
}

Received January 15, 2018; revised July 9, 2018; accepted July 9, 2018

\begin{abstract}
Grid approximation of the Cauchy problem on the interval $\bar{D}=\{0 \leq$ $x \leq d\}$ is first studied for a linear singularly perturbed ordinary differential equation of the first order with a perturbation parameter $\varepsilon$ multiplying the derivative in the equation where the parameter $\varepsilon$ takes arbitrary values in the half-open interval $(0,1]$. In the Cauchy problem under consideration, for small values of the parameter $\varepsilon$, a boundary layer of width $\mathcal{O}(\varepsilon)$ appears on which the solution varies by a finite value. It is shown that, for such a Cauchy problem, the solution of the standard difference scheme on a uniform grid does not converge $\varepsilon$-uniformly in the maximum norm; convergence occurs only under the condition $h \ll \varepsilon$, where $h=d N^{-1}, N$ is the number of grid intervals, $h$ is the grid step-size. Taking into account the behavior of the singular component in the solution, a special piecewise-uniform grid is constructed that condenses in a neighborhood of the boundary layer. It is established that the standard difference scheme on such a special grid converges $\varepsilon$-uniformly in the maximum norm at the rate $\mathcal{O}\left(N^{-1} \ln N\right)$. Such a scheme is called a robust one.

For a model Cauchy problem for a singularly perturbed ordinary differential equation, standard difference schemes on a uniform grid (a classical difference scheme) and on a piecewise-uniform grid (a special difference scheme) are constructed and investigated. The results of numerical experiments are given, which are consistent with theoretical results.
\end{abstract}

Keywords: singularly perturbed Cauchy problem, ordinary differential equation, boundary layer, a priori estimates, standard difference scheme, uniform grid, piecewise-uniform grid, maximum norm, solution decomposition, robust difference scheme.

AMS Subject Classification: 65L05; 65L11; 65L12; 65L70.

Copyright (c) 2018 The Author(s). Published by VGTU Press

This is an Open Access article distributed under the terms of the Creative Commons Attribution License (http://creativecommons.org/licenses/by/4.0/), which permits unrestricted use, distribution, and reproduction in any medium, provided the original author and source are credited. 


\section{Introduction}

The Cauchy problem for regular equations was investigated, e.g., in well known works $[3,4,5,7,9,12]$, but for singularly perturbed equations it has not been considered previously. In this paper, we consider a new class of singularly perturbed problems, namely, the Cauchy problem for a linear singularly perturbed ordinary first-order differential equation with a small parameter $\varepsilon$ multiplying the derivative in the equation where the parameter $\varepsilon$ takes arbitrary values in the half-open interval $(0,1]$. For small values of the parameter $\varepsilon$, a boundary layer of width $\mathcal{O}(\varepsilon)$ appears on which the solution of this problem varies by a finite value. In the present paper, we study the applicability of traditional standard difference schemes for solving such Cauchy problems. It is shown that the use of standard difference schemes on uniform grids leads to large errors of the grid solutions that makes these classical difference schemes unsuitable for practical calculations. Therefore, for the class of singularly perturbed problems under consideration, it becomes necessary to use special robust difference schemes whose solutions converge independently of the perturbation parameter $\varepsilon$, i.e., $\varepsilon$-uniformly, in the maximum norm.

At present, for wide classes of singularly perturbed boundary value problems with elliptic and ordinary differential equations, and for initial boundary value problems with parabolic equations, special numerical methods based on standard difference schemes on grids condensing in boundary layers have been developed and well studied whose solutions converge $\varepsilon$-uniformly in the maximum norm (see, e.g., [1, 2, 6, 10,11] and the bibliography therein). In these special numerical methods, the grid equations are solved on the simplest piecewise-uniform grids which are known in the literature on numerical methods for singularly perturbed problems for elliptic and parabolic equations as Shishkin grids; see, e.g., [8] and the bibliography therein.

In the present paper, for solving the Cauchy problem for a linear singularly perturbed ordinary differential equation, an approach similar to that used previously for singularly perturbed elliptic and parabolic equations in $[1,2,6,10,11]$, is developed to the construction of special difference schemes convergent $\varepsilon$ uniformly in the maximum norm. As a result, a priori estimates are obtained. On the basis of these estimates, a special difference scheme is constructed and $\varepsilon$-uniform convergence in the maximum norm of the grid solution to this scheme for the Cauchy problem under consideration is justified.

Note that many different methods for solving the Cauchy problem are known for regular ordinary differential equations, including special schemes, (see, for example, $[7,12]$ ). But the study of their application to singularly perturbed problems is a large independent research, since it is required to ensure their applicability, that is, to prove convergence, independent of the perturbation parameter, in the maximum norm for the grid solution.

Content of this paper is as follows. The formulation of the Cauchy problem under consideration and the aim of the research are presented in Section 2. A standard difference scheme and a special difference scheme on a piecewiseuniform grid are considered in Section 4 and 5, respectively. A priori estimates of the solution and its derivatives used in the construction of special difference 
schemes and the justification of their $\varepsilon$-uniform convergence in the maximum norm are derived in Section 3. Numerical experiments on the solution of difference schemes with uniform and piecewise-uniform grids for the model Cauchy problem are performed in Section 6.

\section{Problem formulation. Aim of the research}

On the set

$$
\bar{D}, \quad \text { where } \bar{D}=D \cup \Gamma, \quad D=(0<x \leq d], \quad \Gamma=\{x=0\}
$$

we consider the Cauchy problem for a linearly singularly perturbed ordinary first-order differential equation

$$
\begin{array}{rlrl}
L u(x) \equiv \varepsilon a(x) \frac{d}{d x} u(x)+b(x) u(x) & =f(x), & x \in D, \\
u(x) & =\varphi, & & x \in \Gamma,
\end{array}
$$

and besides, ${ }^{1}$

$$
m \leq a(x), b(x) \leq M, \quad|f(x)| \leq M, \quad x \in \bar{D}, \quad|\varphi| \leq M
$$

the functions $a(x), b(x)$, and $f(x)$ are assumed to be sufficiently smooth on $\bar{D}$, the parameter $\varepsilon$ takes arbitrary values from the half-open interval $(0,1]$.

For small values of the parameter $\varepsilon$, a boundary layer appears in the solution of problem $(2.2),(2.1)$ that is a narrow subregion of width $\mathcal{O}(\varepsilon)$ adjacent to the set $\Gamma$ on which the solution of the problem varies by a finite value. By this reason, the standard difference scheme on a uniform grid does not converge $\varepsilon$-uniformly.

Our aim is for Cauchy problem (2.2), (2.1) for a linear singularly perturbed ordinary differential equation, to study applicability of standard difference schemes for solving such problems; to construct and investigate a special difference scheme convergent $\varepsilon$-uniformly in the maximum norm, i.e., robust difference scheme.

\section{A priori estimates of the solution and its derivatives for problem (2.2), (2.1)}

Here we derive a number of a priori estimates for the solution of problem (2.2), (2.1) and its derivatives that are used to construct difference schemes and justify their convergence.

For Cauchy problem (2.2), (2.1) for a linear singularly perturbed ordinary differential equation, a maximum principle is valid (see, e.g., [9] for regular problems).

\footnotetext{
${ }^{1}$ By $M(m)$ we denote sufficiently large (small) positive constants independent of $\varepsilon$. In the case of grid problems, these constants also do not depend on the stencils of the difference schemes.
} 
Lemma 1. Let the data of the Cauchy problem (2.2), (2.1) satisfy the condition

$$
L u(x) \geq 0, \quad x \in D ; \quad u(x) \geq 0, \quad x \in \Gamma .
$$

Then for its solution, the following estimate is valid: $u(x) \geq 0, x \in \bar{D}$.

For the solution of problem (2.2), (2.1), with using the maximum principle, we obtain the estimate

$$
|u(x)| \leq M, \quad x \in \bar{D},
$$

where $M=M_{(3.1)}=\max _{x \in \bar{D}}\left\{b^{-1}(x)|f(x)|\right\}+|\varphi|$.

Taking into account the estimate (3.1), the following "rough" estimate of derivatives is established:

$$
\left|\frac{d^{k}}{d x^{k}} u(x)\right| \leq M \varepsilon^{-k}, \quad x \in \bar{D}, \quad k \leq 2 .
$$

The following theorem holds

Theorem 1. Let the data of the Cauchy problem (2.2), (2.1) satisfy the condition $a, b, f \in C^{1}(\bar{D})$. Then for the solution of this problem and its derivatives, the estimates (3.1) and (3.2) are valid, respectively.

To derive more "fine" a priori estimates of the solution and derivatives, we represent the solution of the Cauchy problem (2.2), (2.1) as the following decomposition $^{2}$

$$
u(x)=U(x)+V(x), \quad x \in \bar{D} .
$$

Here $U(x)$ and $V(x)$ are the regular and singular components of the solution. The regular component $U(x)$ is a sufficiently smooth solution of an inhomogeneous differential equation; its first-order derivative is $\varepsilon$-uniformly bounded. The singular component $V(x)$ which is a boundary layer function, is a solution of a homogeneous equation with a non-homogeneous initial condition; its derivatives are not $\varepsilon$-uniformly bounded.

The function $U(x), x \in \bar{D}$, is represented as the following "expansion":

$$
U(x)=U_{0}(x)+v_{U_{0}}(x), \quad x \in \bar{D},
$$

where $U_{0}(x)$ and $v_{U_{0}}(x)$ are main and "remainder" members in the "expansion" (3.4) with respect to the parameter $\varepsilon$. The function $U_{0}(x)$ is the solution of the degenerate problem

$$
b(x) U_{0}(x)=f(x), \quad x \in \bar{D},
$$

the function $v_{U_{0}}(x), x \in \bar{D}$, is the solution of the problem with a "small" right-hand side

$$
L v_{U_{0}}(x)=-\varepsilon a(x) \frac{d}{d x} U_{0}(x), \quad x \in D, \quad v_{U_{0}}(x)=0, \quad x \in \Gamma .
$$

\footnotetext{
2 Solution decomposition of such a type is known in literature on numerical methods for singularly perturbed problems for elliptic and parabolic equations as the Shishkin decomposition or $S$-decomposition; see, e.g., $[8,11]$ and the bibliography therein.
} 
The function $V(x)$ is the solution of the problem

$$
L V(x)=0, \quad x \in D, \quad V(x)=\varphi-U(0), \quad x \in \Gamma .
$$

Taking into account the following estimates of components in the "expansion" (3.4):

$$
\left|\frac{d^{k}}{d x^{k}} U_{0}(x)\right| \leq M, \quad\left|\frac{d^{k}}{d x^{k}} v_{U_{0}}(x)\right| \leq M \varepsilon^{1-k}, \quad x \in \bar{D}, \quad k \leq 2,
$$

we obtain the following estimates for the components in the decomposition $(3.3)$ :

$$
\begin{aligned}
\left|\frac{d^{k}}{d x^{k}} U(x)\right| & \leq M\left(1+\varepsilon^{1-k}\right), \\
\left|\frac{d^{k}}{d x^{k}} V(x)\right| & \leq M \varepsilon^{-k} \exp \left\{-m \varepsilon^{-1} x\right\}, \quad x \in \bar{D}, \quad k \leq 2,
\end{aligned}
$$

where $m \leq m_{0}, m_{0}=\min _{x}\left\{a^{-1}(x) b(x)\right\}, x \in \bar{D}$.

The following theorem holds

Theorem 2. Let the data of the Cauchy problem (2.2), (2.1) satisfy the condition $a, b, f \in C^{2}(\bar{D})$. Then for the regular and singular components of the solution in the decomposition (3.3), the estimates (3.5) are valid.

\section{Standard difference scheme for problem (2.2), (2.1)}

Consider a standard difference scheme for Cauchy problem (2.2), (2.1) constructed on the basis of a monotone grid approximation of a differential equation (see, e.g., [9] in the case of a regular equation).

\subsection{Construction of a standard difference scheme}

On the set $\bar{D}$ we introduce the grid

$$
\bar{D}_{h}=D_{h} \cup \Gamma_{h} .
$$

Here $\bar{D}_{h}$ is an arbitrary, in general, non-uniform grid on the interval $\bar{D}$. Set $h^{i}=x^{i}-x^{i-1}, x^{i}, x^{i-1} \in \bar{D}_{h}, h=\max _{i} h^{i}, i=1,2, \ldots, N$. Assume that the condition $h \leq M N^{-1}$ holds, where $N+1$ is the number of nodes in the grid $\bar{D}_{h}$.

We approximate problem $(2.2),(2.1)$ by the implicit standard difference scheme:

$$
\Lambda z(x)=f(x), \quad x \in D_{h}, \quad z(x)=\varphi, \quad x \in \Gamma_{h} .
$$

Here $D_{h}=D \cap \bar{D}_{h}, \quad \Gamma_{h}=\Gamma \cap \bar{D}_{h}, x=x^{i}, i=1,2, \ldots, N$,

$$
\Lambda z(x) \equiv \varepsilon a(x) \delta_{\bar{x}} z(x)+b(x) z(x), \quad \delta_{\bar{x}} z(x)=\frac{z\left(x^{i}\right)-z\left(x^{i-1}\right)}{x^{i}-x^{i-1}},
$$

$\delta_{\bar{x}} z(x)$ is the first-order backward difference derivative in $x$.

Difference scheme (4.2), (4.1) is monotone $\varepsilon$-uniformly (for the definition of monotonicity to difference schemes, see, e.g., in [9]). For this scheme, the grid maximum principle is valid. 
Lemma 2. Let for difference scheme (4.2), (4.1), the following condition be satisfied:

$$
\Lambda z(x) \geq 0, \quad x \in D_{h} ; \quad z(x) \geq 0, \quad x \in \Gamma_{h} .
$$

Then the solution $z(x)$ of this difference scheme satisfies the bound

$$
z(x) \geq 0, \quad x \in \bar{D}_{h} .
$$

\subsection{On convergence of standard difference schemes on an arbitrary and uniform grids}

In the case of arbitrary $\operatorname{grid}^{3} \bar{D}_{h(4.1)}$, using a priori estimate (3.2), we obtain the estimate

$$
|u(x)-z(x)| \leq M\left(\varepsilon+N^{-1}\right)^{-1} N^{-1}, \quad x \in \bar{D}_{h(4.1)} .
$$

In the case of the uniform grid

$$
\bar{D}_{h}=\bar{D}_{h}^{u}
$$

we have the estimate similar to (4.3),

$$
|u(x)-z(x)| \leq M\left(\varepsilon+N^{-1}\right)^{-1} N^{-1}, \quad x \in \bar{D}_{h(4.4)}^{u},
$$

where $M_{(4.3)}, M_{(4.5)}=\mathcal{O}(1)$.

Thus, the standard difference schemes (4.2), (4.1) and (4.2), (4.4) converge as $N \rightarrow \infty$ only for fixed values of the parameter $\varepsilon$ with first-order accuracy.

The following theorem holds

Theorem 3. Let the data of the Cauchy problem for the equation (2.2), (2.1) satisfy the condition $(2.2 \mathrm{~b})$, and let the solution of the problem satisfy the estimates from Theorem 1 with $k \leq 2$. Then for the solutions of the standard difference schemes (4.2), (4.1) and (4.2), (4.4), the estimates (4.3) and (4.5) are valid, respectively.

Remark 1. Standard difference schemes (4.2), (4.1) and (4.2), (4.4) converge under the unimprovable condition $N^{-1}=o(\varepsilon)$. According to estimates (4.3) and (4.5), for convergence of schemes (4.2), (4.1) and (4.2), (4.4), it is need to use grids with the number of nodes satisfying the condition $N \gg \varepsilon^{-1}$, i.e., increasing unboundedly as $\varepsilon \rightarrow 0$. Thus, standard difference schemes (4.2), (4.1) and (4.2), (4.4) on an arbitrary and uniform grids, respectively, do not converge $\varepsilon$-uniformly with increasing number of grid nodes.

\section{Special difference scheme for problem (2.2), (2.1) convergent $\varepsilon$-uniformly}

For Cauchy problem (2.2), (2.1), we consider and study a special difference scheme on a piecewise-uniform grid of Shishkin's grid type.

\footnotetext{
3 The notation $\left.D_{(i . j)}\left(L_{(i . j)}, m_{(i . j)}, M_{(i . j)}, D_{h(i . j)}\right)\right)$ means that these sets (operators, constants, grids) were introduced in formula $(i . j)$.
} 


\subsection{Construction of a special difference scheme}

On the set $\bar{D}$ we introduce a special grid

$$
\bar{D}_{h}=\bar{D}_{h}^{s}
$$

here $\bar{D}_{h}^{s}$ is a special piecewise-uniform grid condensing in a neighbourhood of the boundary $\Gamma$, i.e., in the boundary layer region.

When constructing the grid $\bar{D}_{h}^{s}$, we divide the segment $[0, d]$ into two parts $[0, \sigma]$ and $[\sigma, d]$, and, on each of them, we construct uniform meshes with the same number of intervals equal to $2^{-1} N ; N$ is assumed to be even. The value $\sigma$ (transition point) is defined by the relation

$$
\sigma=\sigma(\varepsilon, N)=\min \left[m^{-1} \varepsilon \ln N, 2^{-1} d\right], \quad m=m_{(3.5)} .
$$

Thus, the mesh-sizes in the grid $\bar{D}_{h}^{s}$ are defined as follows:

$$
h_{1}=2 \sigma N^{-1} \quad \text { for } \quad x \in[0, \sigma] ; \quad h_{2}=2(d-\sigma) N^{-1} \text { for } x \in[\sigma, d] \text {. }
$$

For not too small $\varepsilon$, namely, under the condition

$$
\varepsilon \geq 2^{-1} m_{(5.1)} d \ln ^{-1} N
$$

the piecewise-uniform grid $\bar{D}_{h(5.1)}^{s}$ becomes uniform.

We call the standard difference scheme (4.2) on the special piecewise-uniform grid (5.1) (the scheme (4.2), (5.1)) a special difference scheme.

\subsection{On convergence of the special difference scheme}

For the solution of the constructed special difference scheme (4.2), (5.1), taking into account a priori estimates (3.5), and using the grid maximum principle, we obtain the estimate

$$
|u(x)-z(x)| \leq M N^{-1} \ln N, \quad x \in \bar{D}_{h(5.1)} .
$$

Thus, the special difference scheme (4.2), (5.1), i.e., a standard scheme on a piecewise-uniform grid of Shishkin's grid type, converges $\varepsilon$-uniformly in the maximum norm with first-order accuracy up to a logarithmic factor, and it is a robust scheme.

The following theorem holds

Theorem 4. Let for the solution of Cauchy problem (2.2), (2.1), the a priori estimates (3.5) are valid. Then the solution of difference scheme (4.2), (5.1) converges $\varepsilon$-uniformly in the maximum norm with the estimate (5.2).

\section{Numerical study of difference schemes for a model Cauchy problem}

We are interested in the behavior of errors in the grid solutions of the Cauchy problem (2.2), (2.1) for a linearly singularly perturbed ordinary differential 
equation depending on the number of grid nodes and the parameter $\varepsilon$. For this, we investigate the solutions of the standard difference scheme (4.2) on the uniform grid (4.4), and also on the special piecewise-uniform grid (5.1).

The solution of difference scheme (4.2) on arbitrary grid (4.1) is written out in the recurrent form:

$$
\begin{aligned}
\left\{\frac{\varepsilon a}{h_{i-1}}+b\right\} z\left(x_{i}\right) & =\frac{\varepsilon a}{h_{i-1}} z\left(x_{i-1}\right)+f\left(x_{i}\right), \quad h_{i-1}=x_{i}-x_{i-1}, \quad 0<i \leq N \\
z\left(x_{i}\right) & =\varphi, \quad i=0 . \quad x^{i}, x^{i-1} \in \bar{D}_{h} .
\end{aligned}
$$

For computations, it is convenient to represent this solution in the form, resolved with respect to $z\left(x_{i}\right)$

$$
\begin{aligned}
& z\left(x_{i}\right)=\frac{\varepsilon a}{\varepsilon a+b h_{i-1}} z\left(x_{i-1}\right)+f\left(x_{i}\right) \frac{h_{i-1}}{\varepsilon a+b h_{i-1}}, \quad 0<i \leq N \\
& z\left(x_{i}\right)=\varphi, \quad i=0 . \quad x^{i}, x^{i-1} \in \bar{D}_{h} .
\end{aligned}
$$

For the Cauchy model problem, we consider the simplest case when for differential equation (2.2) and the corresponding grid equation (4.2), as well as the calculated formula $(6.1 \mathrm{~b})$, the following condition is satisfied:

$$
a=b=1, \quad f(x)=1, \quad x \in D ; \quad \varphi=0 .
$$

In this case, the exact solution of the differential problem is written out in the following explicit form: $u_{\varepsilon}(x)=1-e^{-x / \varepsilon}, x \in \bar{D}$.

Numerical experiments are performed using techniques similar to that in $[1,2]$. We investigate the behavior of quantities

$$
\begin{aligned}
& E(\varepsilon, N)=\max _{\bar{D}_{h}}\left|u_{\varepsilon}(x)-z_{\varepsilon}(x)\right|, \quad x=x_{i}, \quad x_{i} \in D_{h}, \\
& \bar{E}(N)=\max _{\varepsilon} E(\varepsilon, N)
\end{aligned}
$$

depending on values of the parameter $\varepsilon, \varepsilon=2^{0} \div 2^{-14}$, for $N=2^{2} \div 2^{12}$. Here $z_{\varepsilon}(x)$ is the solution of difference scheme $(4.2),(6.2)$ on the arbitrary grid $\bar{D}_{h(4.1)}$ with number of intervals equal to $N$, and $u_{\varepsilon}(x)$ is the exact solution in nodes $x$ of the corresponding grid. The quantity $E(\varepsilon, N)$ is the maximum pointwise error as a function of $\varepsilon$ and $N$. The quantity $\bar{E}(N)$ is the maximum value with respect to $\varepsilon$ from $E(\varepsilon, N)$.

In numerical experiments, we use as an arbitrary grid either the uniform grid (4.4), or the special piecewise-uniform grid (5.1): the maximum point-wise errors $E(\varepsilon, N)$ and $\bar{E}(N)$ in the solutions of the standard scheme (4.2) on the uniform grid (4.4) and on the special piecewise-uniform grid (5.1) are given in Tables 1 and 2, respectively.

From the Table 1 , one can see that the error $E(\varepsilon, N)$ in the grid solution $z(x)$ of the standard scheme on the uniform grid decreases with increasing $N$ only under the condition $N \gg \varepsilon^{-1}, \varepsilon \in(0,1]$, (that is, above the selected in bold diagonal). Thus, the solution $z(x)$ of the standard difference scheme on the uniform grid converges to the exact solution $u(x)$ only for fixed values of 
Table 1. Maximum point-wise errors $E(\varepsilon, N)$ and $\bar{E}(N)$ in the solution of the standard scheme (4.2) on the uniform grid (4.4)

\begin{tabular}{lllllll}
\hline$\varepsilon \backslash N$ & 4 & 16 & 64 & 256 & 1024 & 4096 \\
\hline 1 & $4.172 \mathrm{e}-2$ & $1.121 \mathrm{e}-2$ & $2.855 \mathrm{e}-3$ & $7.173 \mathrm{e}-4$ & $1.796 \mathrm{e}-4$ & $4.490 \mathrm{e}-5$ \\
$2^{-2}$ & $1.321 \mathrm{e}-1$ & $4.172 \mathrm{e}-2$ & $1.121 \mathrm{e}-2$ & $2.855 \mathrm{e}-3$ & $7.173 \mathrm{e}-4$ & $1.796 \mathrm{e}-4$ \\
$2^{-4}$ & $\mathbf{1 . 8 1 7 e}-\mathbf{1}$ & $1.321 \mathrm{e}-1$ & $4.172 \mathrm{e}-2$ & $1.121 \mathrm{e}-2$ & $2.855 \mathrm{e}-3$ & $7.173 \mathrm{e}-4$ \\
$2^{-6}$ & $5.882 \mathrm{e}-2$ & $\mathbf{1 . 8 1 7 e}-\mathbf{1}$ & $1.321 \mathrm{e}-1$ & $4.172 \mathrm{e}-2$ & $1.121 \mathrm{e}-2$ & $2.855 \mathrm{e}-3$ \\
$2^{-8}$ & $11.538 \mathrm{e}-2$ & $5.882 \mathrm{e}-2$ & $\mathbf{1 . 8 1 7 e}-\mathbf{1}$ & $1.321 \mathrm{e}-1$ & $4.172 \mathrm{e}-2$ & $1.121 \mathrm{e}-2$ \\
$2^{-10}$ & $3.891 \mathrm{e}-3$ & $1.538 \mathrm{e}-2$ & $5.882 \mathrm{e}-2$ & $\mathbf{1 . 8 1 7 e}-\mathbf{1}$ & $1.321 \mathrm{e}-1$ & $4.172 \mathrm{e}-2$ \\
$2^{-12}$ & $9.756 \mathrm{e}-4$ & $3.891 \mathrm{e}-3$ & $1.538 \mathrm{e}-2$ & $5.882 \mathrm{e}-2$ & $\mathbf{1 . 8 1 7 e}-\mathbf{1}$ & $1.321 \mathrm{e}-1$ \\
$2^{-14}$ & $2.441 \mathrm{e}-4$ & $9.756 \mathrm{e}-4$ & $3.891 \mathrm{e}-3$ & $1.538 \mathrm{e}-2$ & $5.882 \mathrm{e}-2$ & $\mathbf{1 . 8 1 7 e}-\mathbf{1}$ \\
\hline$\overline{\mathbf{E}}(\mathbf{N})$ & $\mathbf{1 . 8 1 7 e}-\mathbf{1}$ & $\mathbf{1 . 8 1 7 e}-\mathbf{1}$ & $\mathbf{1 . 8 1 7 e}-\mathbf{1}$ & $\mathbf{1 . 8 1 7 e}-\mathbf{1}$ & $\mathbf{1 . 8 1 7 e}-\mathbf{1}$ & $\mathbf{1 . 8 1 7 e}-\mathbf{1}$ \\
\hline
\end{tabular}

Table 2. Maximum point-wise errors $E(\varepsilon, N)$ and $\bar{E}(N)$ in the solution of the standard scheme (4.2) on the special piecewise-uniform grid (5.1)

\begin{tabular}{lllllll}
\hline$\varepsilon \backslash N$ & 4 & 16 & 64 & 256 & 1024 & 4096 \\
\hline 1 & $4.172 \mathrm{e}-2$ & $1.121 \mathrm{e}-2$ & $2.855 \mathrm{e}-3$ & $7.173 \mathrm{e}-4$ & $1.796 \mathrm{e}-4$ & $4.490 \mathrm{e}-5$ \\
$2^{-2}$ & $1.321 \mathrm{e}-1$ & $4.172 \mathrm{e}-2$ & $1.121 \mathrm{e}-2$ & $2.855 \mathrm{e}-3$ & $7.173 \mathrm{e}-4$ & $1.796 \mathrm{e}-4$ \\
$2^{-4}$ & $1.691 \mathrm{e}-1$ & $9.883 \mathrm{e}-2$ & $4.172 \mathrm{e}-2$ & $1.121 \mathrm{e}-2$ & $2.855 \mathrm{e}-3$ & $7.173 \mathrm{e}-4$ \\
$2^{-6}$ & $\mathbf{1 . 6 9 1 e}-\mathbf{1}$ & $\mathbf{9 . 8 8 3 e}-\mathbf{2}$ & $\mathbf{4 . 3 2 9 e}-\mathbf{2}$ & $\mathbf{1 . 5 3 9 e}-\mathbf{2}$ & $\mathbf{4 . 9 2 5 e}-\mathbf{3}$ & $\mathbf{1 . 4 8 9 e}-\mathbf{3}$ \\
$2^{-8}$ & $1.691 \mathrm{e}-1$ & $9.883 \mathrm{e}-2$ & $4.329 \mathrm{e}-2$ & $1.539 \mathrm{e}-2$ & $4.925 \mathrm{e}-3$ & $1.489 \mathrm{e}-3$ \\
$\ldots$ & $\ldots$ & $\ldots$ & $\ldots$ & $\ldots$ & $\ldots$ \\
$2^{-18}$ & $1.691 \mathrm{e}-1$ & $9.883 \mathrm{e}-2$ & $4.329 \mathrm{e}-2$ & $1.539 \mathrm{e}-2$ & $4.925 \mathrm{e}-3$ & $1.489 \mathrm{e}-3$ \\
\hline$\overline{\mathbf{E}}(\mathbf{N})$ & $\mathbf{1 . 6 9 1 e}-\mathbf{1}$ & $\mathbf{9 . 8 8 3 e}-\mathbf{2}$ & $\mathbf{4 . 3 2 9 e}-\mathbf{2}$ & $\mathbf{1 . 5 3 9 e}-\mathbf{2}$ & $\mathbf{4 . 9 2 5 e}-\mathbf{3}$ & $\mathbf{1 . 4 8 9 e}-\mathbf{3}$ \\
\hline
\end{tabular}

the parameter $\varepsilon$ and it does not converge $\varepsilon$-uniformly (see $\bar{E}(N)$ in the last row).

From the Table 2 it is seen that the error $E(\varepsilon, N)$ in the grid solution $z(x)$ of the special difference scheme decreases with increasing $N$ for each value of the parameter $\varepsilon$; moreover, the value of this error does not change for $\varepsilon$ from $2^{-6}$ to $2^{-18}$ for each $N$, that is, the error is independent of the parameter $\varepsilon$ (this error is "set"). Thus, the solution $z(x)$ of the special difference scheme, that is, the standard scheme on the piecewise-uniform grid, converges to the exact solution $u(x) \varepsilon$-uniformly (see $\bar{E}(N)$ in the last row).

Figure 1 shows the exact solution of the differential problem and the numerical solution of the standard difference scheme with "zoom" in the domain by $x$ from 0.0 to 0.25 .

Figure 2 shows the exact solution of the differential problem and the numerical solution of the special difference scheme with "zoom" in the domain by $x$ from 0.0 to 0.06 . 


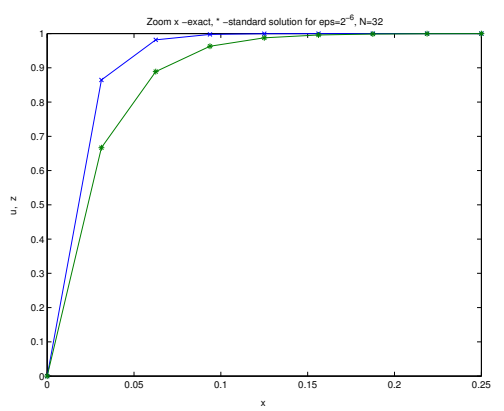

Figure 1. Zoom $\times$ - exact solution, $*$ - standard solution for $\varepsilon=\mathbf{2}^{-\mathbf{6}}, \boldsymbol{N}=\mathbf{3 2}$

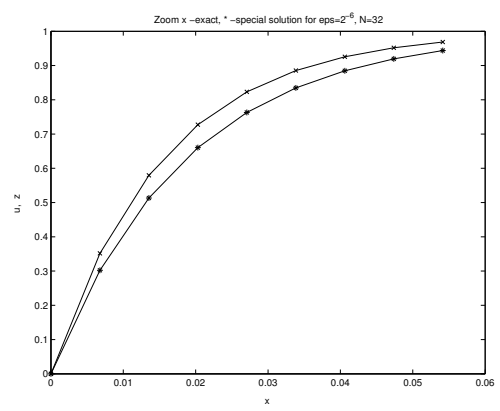

Figure 2. Zoom $\times$ - exact solution, $*$ - special solution for $\varepsilon=\mathbf{2}^{-\mathbf{6}}, \mathbf{N}=\mathbf{3 2}$

\section{Conclusions}

Grid approximation of the Cauchy problem on the interval $\bar{D}=\{0 \leq x \leq d\}$ have been considered for a linear singularly perturbed ordinary first-order differential equation.

For the Cauchy problem under study, the following results are obtained:

- A priori estimates of the solution and its regular and singular components are obtained (see the statement of Theorem 2 in Section 3).

- It is shown that the use of classical difference schemes, i.e., standard difference schemes on uniform grids leads to the errors of the grid solutions of order $\mathcal{O}(1)$ (see the statement of Theorem 3 in Section 4).

- Taking into account the behaviour of the singular component in the solution, a special piecewise-uniform grid of Shishkin's grid type is constructed that condenses in a neighbourhood of the boundary layer.

- Using the a priori estimates, it is proved that the special difference scheme on the piecewise-uniform Shishkin's grid, converges $\varepsilon$-uniformly in the maximum norm at the rate $\mathcal{O}\left(N^{-1} \ln N\right)$ (see the statement of Theorem 4 in Section 5), i.e., it is a robust grid, while the classical difference scheme does not converge $\varepsilon$-uniformly and it converges only for fixed values of the parameter $\varepsilon$. 
- Numerical study of standard difference schemes on uniform and piecewiseuniform grids is performed for a model Cauchy problem. The results of numerical experiments are in agreement with the theoretical ones.

\section{Acknowledgements}

This research was partially supported by the Russian Foundation for Basic Research under grant No.16-01-00727. The authors are grateful to Corresponding Member of Russian Academy of Science V.E. Tretyakov for attention to this research and helpful discussion of results obtained.

\section{References}

[1] P.W. Hemker, G.I. Shishkin and L.P. Shishkina. The use of defect correction for the solution of parabolic singular perturbation problems. ZAMM-Z. Angew. Math. Mech., 77(1):59-74, 1997. https://doi.org/10.1002/zamm.19970770111.

[2] P.W. Hemker, G.I. Shishkin and L.P. Shishkina. $\varepsilon$-uniform schemes with highorder time-accuracy for parabolic singular perturbation problems. IMA J. Numer. Anal., 20(1):99-121, 2000. https://doi.org/10.1093/imanum/20.1.99.

[3] N.N. Kalitkin. Numerical methods. Nauka, Moscow, 1978. (in Russian)

[4] N.N. Kalitkin and P.V. Koriakin. Numerical methods. Methods of mathematical physics. Akademia, Moscow, 2013. (in Russian)

[5] G.I. Marchuk and V.V. Shaidurov. Difference Methods and Their Interpolations. Springer-Verlag, New York, 1983.

[6] J.J.H. Miller, E. O'Riordan and G.I. Shishkin. Fitted numerical methods for singular perturbation problems. Error estimates in the maximum norm for linear problems in one and two dimensions, Revised Edition. World Scientific, Singapore, 2012.

[7] A. Quarteroni, R. Sacco and F. Saleri. Numerical Mathematics, Second Edition. Springer, Berlin Heidelberg, 2007.

[8] H.-G. Roos, M. Stynes and L. Tobiska. Numerical Methods for Singularly Perturbed Differential Equations. Convection-Diffusion-Reaction and Flow Problems. Second Edition. Marcel Dekker, Springer-Verlag, Berlin, 2008.

[9] A.A. Samarskii. The Theory of Difference Schemes. Marcel Dekker, New York, 2001 .

[10] G.I. Shishkin. Discrete Approximations of Singularly Perturbed Elliptic and Parabolic Equations. Russian Academy of Sciences, Ural Section, Ekaterinburg, 1992. (in Russian)

[11] G.I. Shishkin and L.P Shishkina. Difference Methods for Singular Perturbation Problems. Monographs \& Surveys in Pure \& Applied Mathematics. Chapman and Hall/CRC, Boca Raton, 2009.

[12] E. Süli and D. Mayers. An Introduction to Numerical Analysis. Cambridge University Press, Cambridge, 2003. 